\title{
Embedded System Design for Vehicle Semi-active Suspension System
}

\author{
Wenjun $\mathrm{Li}^{1, \mathrm{a}}$, Hongkun Zhang ${ }^{2, \mathrm{~b}^{*}}$ \\ ${ }^{1}$ Transportation College, Jilin University, No.5988 Renmin Street Changchun 130025, China \\ ${ }^{2}$ State Key Laboratory of Automotive Simulation and Control, Jilin University, No.5988 Renmin \\ Street, Changchun 130025, China \\ aemail: liwj@jlu.edu.cn, bemail: zhanghk@jlu.edu.cn \\ ${ }^{*}$ Corresponding author: Hongkun Zhang
}

\begin{abstract}
Keywords: Embedded System, microcontroller, suspension system, control strategy, Hardware-in-the-loop Simulation.

Abstract. Vehicle semi-active suspension has been more and more widely used since it can provide good operation stability and ride comfort. This paper focuses on embedded system design based on Infineon C164CI microcontroller for vehicle semi-active suspension. The hardware design of electronic suspension control unit (ESCU) is introduced in detail. The integrated control strategy for semi-active suspension system is proposed. The test bench of hardware-in-the-loop simulation (HILS) is built and the functions of ESCU are verified. The HILS simulation results show that the performances of ESCU achieve design requirement and the integrated control strategy is rational and effective.
\end{abstract}

\section{Introduction}

Vehicle suspension system can be roughly divided into three categories, that is, passive suspension, semi-active suspension and active suspension. The purpose of suspension system control is to improve road handling, ride comfort and operation stability of vehicles. Compared with active and passive suspension systems, semi-active suspension system has the advantages of the other two types, i.e. it provides better performances than passive suspension and is more economical and safer than active suspension [1]. Consequently, Semi-active suspension systems are getting more attention in the development of suspension system. In this paper, electronic suspension control unit (ESCU) based on Infineon microcontroller- $\mathrm{C} 164 \mathrm{CI}$ is designed. The integrated control strategy which integrates Skyhook and MiniMax strategies is proposed and the models of the strategy are established in Matlab/Simulink. Performances of semi-active suspension controlled by the integrated strategies and passive suspension are compared and evaluated by HILS test system and the simulation results are analyzed. Conclusions are given in the end of the paper.

\section{Hardware Design of ESCU Based on C164CI}

In our design, Infineon microcontroller named C164CI is selected as the main chip of ESCU. C164CI is a powerful 16-bit device composed of standard on-chip peripherals including a 16-bit CPU with 4-Stage Pipeline, 64K bytes on-chip ROM or program flash, 2 KBytes On-Chip Internal RAM and 2 KBytes On-Chip Extension RAM , 8-Channel 10-bit A/D Converter with Programm (Conversion Time down to $7.8 \mu \mathrm{s}$ ), Capture/Compare Unit for flexible PWM Signal Generation, On-Chip CAN Interface with 15 Message Objects and so on. It can meet the control requirement of our control system. ESCU mainly consists of master chip C164CI, input signal condition circuit, output signal drive circuit and power supply circuit. Input signals include vertical body accelerometer, wheel accelerometer, wheel load force sensor and spring displacement sensor. These signals are processed by signal conditioner and transmitted to analog-to-digital converter of C164CI. ESCU can communicate with other electric control units on vehicle by using CAN module integrated in C164CI to obtain body velocity. According to the sensors input signals and the control algorithm the PWM output signals are produced by Capture/Compare Unit C164CI. PWM signals are amplified by drive 
circuit to control solenoid valve damper in order to adjust damping of semi-active suspension system. The block diagram of ESCU is shown in Fig. 1.

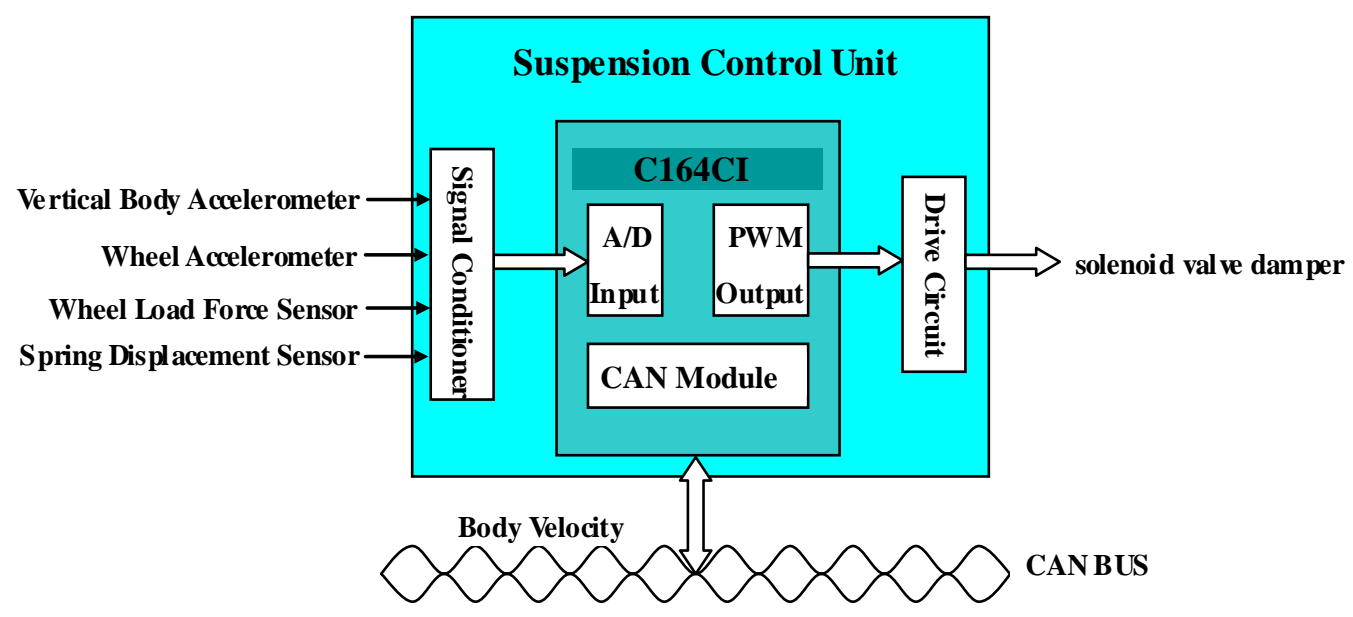

Fig. 1. Block Diagram of SCU

\section{Integrated Control Strategy for Vehicle Semi-active Suspension}

\section{A. A QUARTER-CAR SEMI-ACTIVE SUSPENSION SYSTEM MODEL}

As we know that the ride characteristics of passenger car can be characterized by considering the 'quarter-car' model [4]. This method has been widely applied to investigate the performances of passive [5], semi-active [6] and active-suspension. The quarter car semi-active suspension system model is shown in Fig. 2.

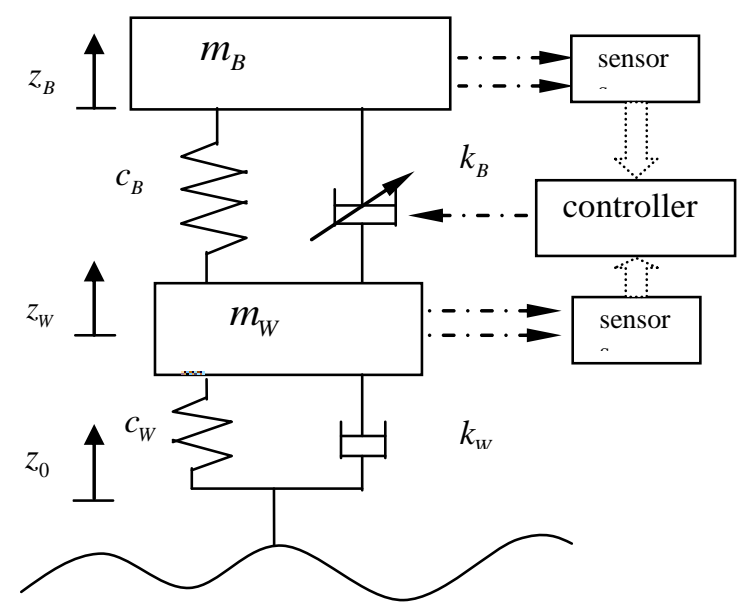

Fig. 2. 2 DOF semi-active suspension system model

Here, the model considers the body mass $m_{B}$, wheel mass $m_{w}$, wheel vertical stiffness $c_{w}$, body vertical stiffness $c_{B}$ and damping coefficient of wheel damper $k_{w}$. It has two degrees of freedom, body deflection $z_{B}$, wheel deflection $z_{w}$, and is excited by $z_{0}$. The model is governed in analytical form by the following equation.

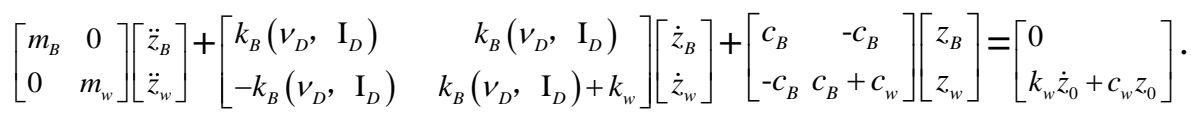

According to above equation the linear differential equations describing the dynamics of the semi-active suspension can be written as: 


$$
\begin{aligned}
& m_{B} \ddot{z}_{B}=-k_{B}\left(\dot{z}_{B}-\dot{z}_{w}\right)-c_{B}\left(z_{B}-z_{w}\right) . \\
& m_{w} \ddot{z}_{w}=k_{B}\left(\dot{z}_{B}-\dot{z}_{w}\right)-k_{w}\left(\dot{z}_{w}-\dot{z}_{0}\right)+c_{B}\left(z_{B}-z_{w}\right)-c_{w}\left(z_{w}-z_{0}\right) .
\end{aligned}
$$

Quarter car model can be established according to equations (2) (3) using MATLAB/Simulink.

\section{B. Integrated Control Strategy of Semi-active suspension}

In this paper, we integrate Skyhook and MiniMax control strategy to control semi-active suspension system.

\section{(1) Skyhook Control Stategy}

Here, we use on-off skyhook control to improve the ride comfort. The control law can be described as follows:

$$
c_{s k y}=\left\{\begin{array}{l}
c_{\max } \text { if } v_{r} v_{b} \geq 0 \\
c_{\min } \text { otherwise }
\end{array} .\right.
$$

Where, $v_{b}$ is the absolute velocity of the body and $v_{r}$ is the relative velocity of the body and wheel across the suspension. The control law indicates that a maximum damping force should be applied to reduce the body acceleration if the relative velocity of the body with respect to the wheel is in the same direction as that of the body velocity. On the other hand, if the two velocities are in the opposite directions, the damping force should be at a minimum value to minimize body acceleration.

\section{(2) MiniMax Control Strategy}

It is very important to reduce wheel load variations in order to keep safety of vehicle in semi-active suspension system. A Wheel Load Influence Matrix can be induced by considering every possible state of damper movement. The matrix is shown in Table 1.

Table 1 Wheel Load Influence Matrix

\begin{tabular}{|l|l|l|}
\hline $\begin{array}{c}\text { Shock abs. } \\
\text { Switching stage } \\
\text { of shock abs. }\end{array}$ & Compression & Rebound \\
\hline Hard to soft & $\begin{array}{l}\text { Decrease in } \\
\text { wheel load }\end{array}$ & $\begin{array}{l}\text { Increase in } \\
\text { wheel load }\end{array}$ \\
\hline Soft to hard & $\begin{array}{l}\text { Increase in } \\
\text { wheel load }\end{array}$ & $\begin{array}{l}\text { Decrease in } \\
\text { wheel load }\end{array}$ \\
\hline
\end{tabular}

We can understand the Matrix as following manner: in rebound, if the damper is switched from hard to soft, this will lead to a increase in wheel load. In compression, if the damper is switched from hard to soft, this will lead to a decrease in wheel load. It is possible to purposely influence the course of wheel load according to the matrix. This knowledge about the effect of switching the damper can be implemented into a switching logic. This logic chooses between the two characteristic lines 'soft' and 'hard', depending on the inputs 'damper velocity' and 'request of wheel load', and is therefore called MiniMax-controller.

In Fig.3, the principle function of the MiniMax controller is shown. Depending on the inputs $v_{D}$ and $F_{z, \text { req }}$ the damper current $I_{D}$ is switched to either 0 or 1.6A (hard or soft damper). This means if either the request of wheel load or the damper velocity changes its sign, the damper will be switched from hard to soft or vice versa. The damper velocity $v_{D}$ or the sign of the damper velocity sign $\left(v_{D}\right)$ provides with information about the direction into which damper is moving compression or rebound. 
The request of wheel load $F_{z, \text { req }}$ has values of either one (increase of wheel load) or minus one (decrease of wheel load).

We propose that Skyhook and MiniMax strategies are integrated to control semi-active suspension system to improve ride comfort and drive safety respectively in different working condition.

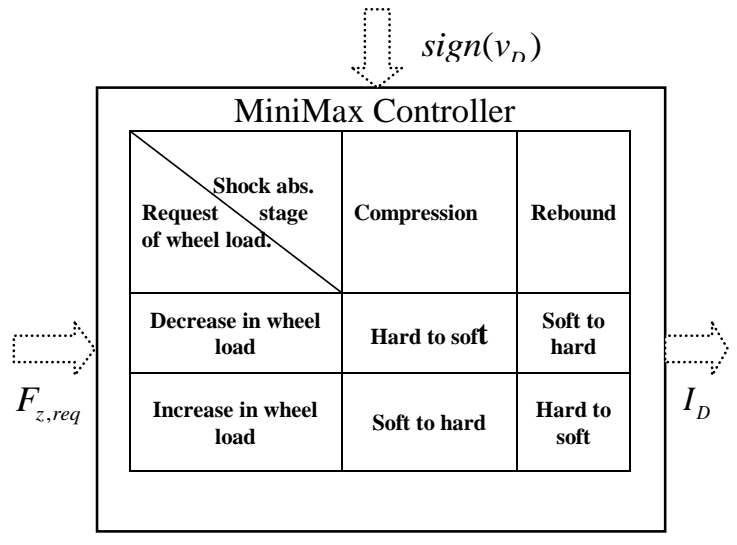

Fig. 3. MiniMax Controller

\section{HILS AND SIMULATION RESULTS}

In our HILS system, Matlab/Simulink is used as a computer aided-control system tool [3] for modeling the quarter car parameters, passive system, skyhook and MiniMax control algorithm. A host PC is used to implement quarter car semi-active suspension model. The processor DS1005 PPC board, multi-Channel A/D Board DS2003 and CAN interface board DS4302 are integrated in the AutoBox. In addition, our HILS test bench also includes the actual ESCU and its peripheral hardware, real sensors and actuator (Damper) and the virtual vehicle model and control strategies to form a closed loop to be simulated in real time. Fig. 4 shows the HILS configuration. The process of simulation is managed and monitored by ControlDesk of dSPACE.

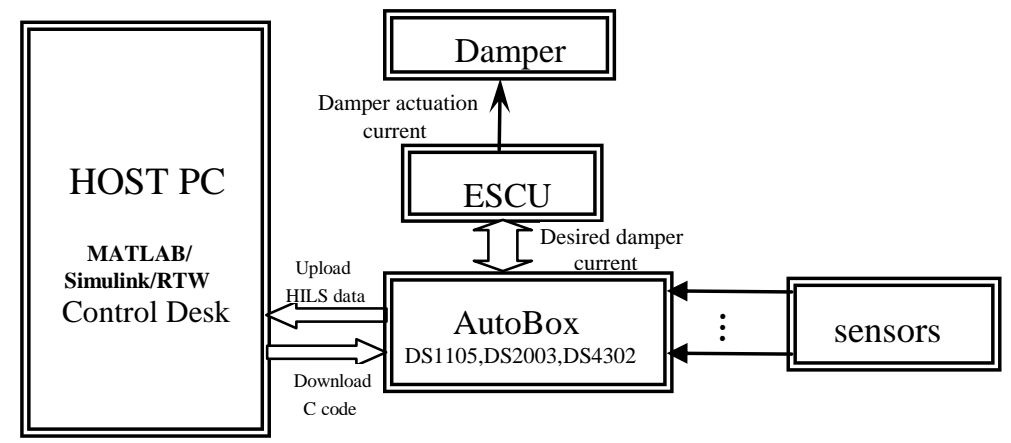

Fig. 4. Configuration for the hardware-in-the-loop simulations

The simulation results are shown in Fig. 5 and Fig. 6. In order to compare the control performances, they were all excited by sine wave that the amplitude is $1 \mathrm{~cm}$. Fig. 5 shows vertical body acceleration response of passive, skyhook and MiniMax control. As can be seen from Fig. 5, skyhook control can achieves more reduction of resonant peak of the body mass than that of passive suspension and MiniMax controller and thus gives a good ride comfort. 


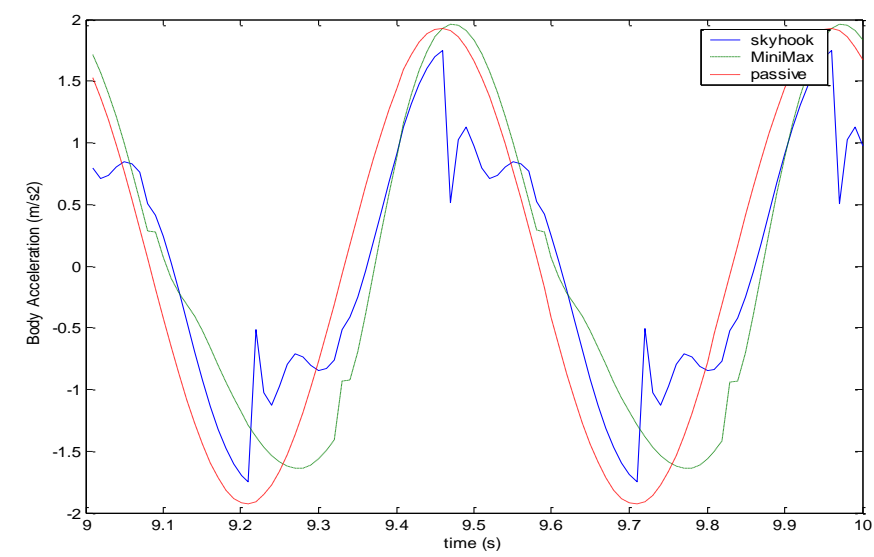

Fig. 5. Comparison of Body Acceleration Response of Passive, Skyhook and MiniMax

Wheel load force of passive, skyhook and MiniMax are shown in Fig. 6. Compared with passive system and skyhook, drive safety can be improved by purposefully increasing or decreasing the wheel load by using MiniMax controller.

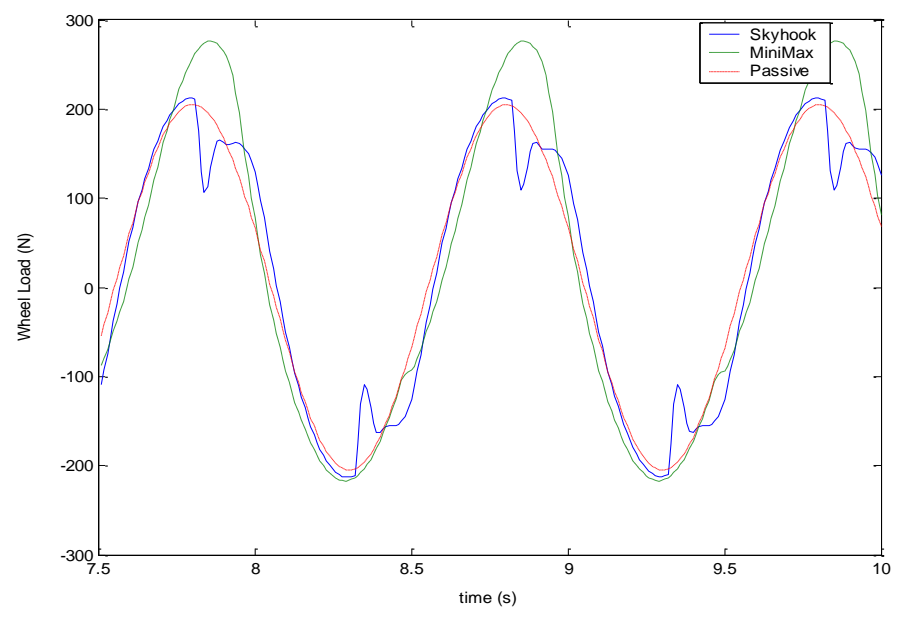

Fig. 6. Comparison of Wheel Load Response of Passive, Skyhook and MiniMax

\section{Conclusion}

In this paper, ESCU based on C164CI microcontroller is designed to fulfill the control functions of semi-active suspension system. The HILS test bench is built and the integrated control strategy is simulated. The results of simulations indicate that the performances of semi-active suspension can be effectively improved by using Skyhook and MiniMax strategies. Consequently, skyhook and MiniMax strategies can be integrated during the vehicle is running in proper moment for effectively improving both riding comfort and safety of vehicle.

\section{Acknowledgements}

This research was financially supported by joint project of university and enterprise.

\section{References}

[1] K. Yi, B.S. Song, A new adaptive sky-hook control of vehicle semi-active suspension. J. Automob. Eng., 213 (3) (1999), pp. 293-303 
[2] Deepa Ramaswamy, A case study in hardware-in-the-loop testing: Development of an ECU for a hybrid electric vehicle (C).SAE Paper 2004-01-0303.

[3] F. Balarin et al., Hardware-Software Co-Design of Embedded Systems - The Polis Approach, Kluwer Academic Publishers, 1997.

[4] Crolla, D. A, Vehicle dynamics: theory into practice. Proc. Inst. Mech. Engrs, Part D: J. Automobile Engineering, 1996, 210, 83-94.

[5] Sharp, R. S. and Hassan, S. A, Evaluation of passive automotive suspension systems with variable stiffness and damping parameters. Veh. System Dynamics, 1986, 15(6), 335-350.

[6] Cebon, D., Besinger, F. H., and Cole, D. J., Control strategies for semi-active lorry suspensions. Proc.Inst. Mech. Engrs, Part D: J. Automobile Engineering, 1996, 210, 161-178. 